\title{
Acute decrease in alkaline phosphatase after brain injury: a potential mechanism for tauopathy
}

Peethambaran Arun*, Samuel Oguntayo, Stephen VanAlbert, Irene Gist, Ying Wang, Madhusoodana P. Nambiar, Joseph B. Long*

Blast-Induced Neurotrauma Branch, Center for Military Psychiatry and Neuroscience, Walter Reed Army Institute of Research, Silver Spring, MD 20910, USA

*Authors for correspondence: 503 Robert Grant Ave, Walter Reed Army Institute of Research, Silver Spring, MD 20910, USA. Phone: +1-301-319-2009/9529;

Fax: +1-301-319-9839.

E-mail: peethambaran.arun.ctr@mail.mil; joseph.b.long.civ@mail.mil

\begin{abstract}
Dephosphorylation of phosphorylated Tau (pTau) protein, which is essential for the preservation of neuronal microtubule assemblies and for protection against traumainduced tauopathy and chronic traumatic encephalopathy (CTE), is primarily achieved in brain by tissue non-specific alkaline phosphatase (TNAP). Paired helical filaments (PHFs) and Tau isolated from Alzheimer's disease (AD) patients' brains have been shown to form microtubule assemblies with tubulin only after treatment with TNAP or protein phosphatase-2A, $2 \mathrm{~B}$ and -1 , suggesting that Tau protein in the PHFs of neurons
\end{abstract}


in $A D$ brain is hyperphosphorylated, which prevents microtubule assembly. Using blast or weight drop models of traumatic brain injury (TBI) in rats, we observed pTau accumulation in the brain as early as $6 \mathrm{~h}$ post-injury and further accumulation which varied regionally by $24 \mathrm{~h}$ post-injury. The $p T a u$ accumulation was accompanied by reduced TNAP expression and activity in these brain regions and a significantly decreased plasma total alkaline phosphatase activity after the weight drop. These results reveal that both blast- and impact acceleration-induced head injuries cause an acute decrease in the level/activity of TNAP in the brain, which potentially contributes to trauma-induced accumulation of $p T a u$ and the resultant tauopathy. The regional changes in the level/activity of TNAP or accumulation of $p$ Tau after these injuries did not correlate with the accumulation of amyloid precursor protein, suggesting that the basic mechanism underlying tauopathy in TBI might be distinct from that associated with AD.

Key words: Blast exposure, head impact/acceleration, tauopathy, chronic traumatic encephalopathy, traumatic brain injury, tissue non-specific alkaline phosphatase

\section{INTRODUCTION}

Advancements in far-forward medical care have greatly increased the survivability of traumatic brain injuries (TBIs), which in recent military conflicts have been primarily attributable to the widespread use of improvised explosive devices and other modern explosive weaponries. In particular, exposure to blast has been described as the major cause of TBI and associated disabilities in the recent wars in Iraq and Afghanistan [19]. Although several biochemical and histopathological changes have been preclinically documented in the central nervous system after blast exposure 
$[6,7,15,18,25,28,32]$, the potentially complex pathophysiological mechanisms triggering long-term neurobehavioral abnormalities are not well understood, which has hampered the development of effective countermeasures and diagnostic approaches.

Recent studies indicate that chronic traumatic encephalopathy (CTE), a tau protein-linked neurodegenerative disorder which has been observed in several athletes with a history of multiple concussions, shares clinical symptoms and neuropathological features described in victims of blast exposure [9]. In particular, phosphorylated Tau ( $p$ Tau) protein neuropathology, with perivascular neurofibrillary degeneration, is recognized as a distinct feature of CTE and has been observed postmortem in the brains of blast victims and contact-sport athletes. Brains of mice exposed to blast overpressure in a shock tube also showed neuropathological features of CTE such as phosphorylated tauopathy, myelinated axonopathy, microvasculopathy, neuroinflammation and neurodegeneration, prompting postulation that blast-induced hyperacceleration of the head may play an important role in the development of CTElike neuropathology [9]. Phosphorylation of Tau protein disrupts microtubule assembly in neurons, which can result in tauopathy and the formation of neurofibrillary tangles seen in neurodegenerative disorders such as Alzheimer's disease (AD) [11, 14, 30]. Dephosphorylation of $p T a u$ is critical to prevent tauopathy and to restore microtubule assembly for neuroregeneration.

By dephosphorylating pTau in neurons, tissue non-specific alkaline phosphatase (TNAP) may play a major role in the etiology of brain disorders involving this neuropathological feature $[11,14,30]$. Paired helical filaments and Tau protein isolated from $A D$ patients' brains were shown to form a microtubule assembly with tubulin in vitro 
only after treatment with alkaline phosphatase or protein phosphatase-2A, $2 \mathrm{~B}$ and -1 , suggesting that Tau protein in the paired helical filaments of neurons in AD brain is hyperphosphorylated, which prevents microtubule assembly [11, 14, 30]. Alkaline phosphatase showed significantly higher activity in dephosphorylating pTau compared to the other protein phosphatases studied [30].

A number of studies indicate that accumulation of amyloid precursor protein (APP) and $\beta$-amyloid peptides induces the phosphorylation of Tau, leading to microtubule disassembly, which is an accepted neuropathological feature of $A D[4,10$, $17,26,27,29,33]$. Activation of mitogen-activated protein kinase by accumulated APP has been described as a mechanism yielding the phosphorylation of Tau protein [10]. In a hybrid septal cell line, treatment with aggregated $\beta$-amyloid peptide resulted in accumulation of $p T a u$ and paired helical filaments and alkaline phosphatase treatment abolished the effect [17], emphasizing the potentially important role of $\beta$-amyloid peptide in triggering and TNAP in preventing Tau phosphorylation.

In the present study, we utilized rat models of blast-induced TBI using a shock tube and impact acceleration-induced TBI using weight drop and explored the acute changes in the expression of $p$ Tau and level/activity of TNAP in different brain regions after these insults. We have also examined whether the alterations in the expression of pTau and TNAP are associated with a corresponding acute change in the expression of APP to examine a possible role of accumulated APP in the initiation and development of tauopathy after TBI. 


\section{EXPERIMENTAL PROCEDURES}

\subsection{Animals and blast injury}

All animal experiments were conducted in accordance with the Animal Welfare Act and other federal statutes and regulations relating to animals and experiments involving animals, and adhered to principles stated in the Guide for the Care and Use of Laboratory Animals (NRC Publication 2011 edition) using an Institutional Animal Care and Use Committee approved protocol. Male Sprague Dawley rats, 9-10 weeks old that weighed 300-350 g (Charles River Laboratories, Wilmington, MA ) were housed at 20$22^{\circ} \mathrm{C}(12 \mathrm{~h} \mathrm{light/dark}$ cycle $)$ with free access to food and water ad libitum. A total of 4 animals/group was used for each time point.

\subsection{Brain injury by blast exposure}

Blast exposure was carried out using a compressed air-driven shock tube as described earlier [5]. Briefly, after $4 \%$ isoflurane gas anesthesia in an induction box for 8 $\min \left(\mathrm{O}_{2}\right.$ flow rate $\left.1.5 \mathrm{~L} / \mathrm{min}\right)$, rats were immediately tautly secured in a transverse prone position in coarse mesh netting $2.5 \mathrm{ft}$ within the mouth of the $15 \mathrm{ft}$ long and $1 \mathrm{ft}$ internal diameter expansion chamber with the right side of the head/body facing the pressure chamber. Rats were exposed to single shockwave (peak static pressure of $19.0 \mathrm{psi}$ $(131 \mathrm{kPa})$ with a $9 \mathrm{msec}$ positive phase duration). Blast overpressure flow conditions were recorded using piezoresistive pressure transducers (Meggit Inc., San Juan Capistrano, CA) mounted in the rat holder which provided measurements of total and side-on pressure waveforms. Sham control animals were included in all individual experiments and were treated in the same fashion without exposure to blast.

\subsection{Brain injury by weight drop}


As originally described by Marmarou et al. [20], the injury device consisted of a vertically secured $2.5 \mathrm{~m}$ long Plexiglas tube with a $19 \mathrm{~mm}$ inner diameter. A Mylar helmet was mounted on the heads of the isoflurane-anesthetized rats to prevent any skull fracture during weight drop. The rats were placed in a prone position on a $12 \times 12$ $\times 43 \mathrm{~cm}$ foam bed (Type E manufactured by Foam to Size, Inc., Ashland, VA). After placing the rat on the foam bed, the bed was positioned directly under the tube. The rat's head and the cap were adjusted so that the striking plate was horizontal and parallel to the impacting face of the falling weight. The animal's body was held lightly on the form during impact. Brain injury was produced by dropping the cylindrical weight $(500 \mathrm{~g})$ from a predetermined height $(150 \mathrm{~cm})$. Rebound impact by the weight was prevented by sliding the foam bed and rat away from the tube immediately after impact/acceleration. As noted above, sham controls were anesthetized and handled without being subjected to brain injury.

\subsection{Sample collection for analyses}

Rats were euthanized 6 and $24 \mathrm{~h}$ post-injury and blood plasma and brain samples were collected. Cortex, hippocampus and brainstem were dissected and pooled across hemispheres over ice, immediately frozen and stored at $-80^{\circ} \mathrm{C}$ along with blood plasma until analyses.

\subsection{Measurement of enzyme activity}

The activity of TNAP in the brain and activity of total alkaline phosphatase in the blood plasma were determined using a diagnostic kit (Randox Laboratories, Kearneysville, WV) according to the manufacturer's instructions. In this assay, the 
colorless $p$-nitrophenyl phosphate is hydrolyzed to yellow colored $p$-nitophenol by alkaline phosphatase at alkaline $\mathrm{pH}$ in the presence of Magnesium ions. The absorbance of the yellow colored $p$-nitrophenol formed, which will be directly proportional to the activity of alkaline phosphatase, was measured at $400 \mathrm{~nm}$. In the case of brain tissue, $10 \%$ homogenates were made in T-PER tissue protein extraction reagent (Pierce Chemical Co, Rockford, IL) containing protease inhibitor cocktail (Sigma-Aldrich, St. Louis, MO) using an ultrasonic homogenizer. The homogenates were centrifuged at $5000 \times g$ for $5 \mathrm{~min}$ at $4^{\circ} \mathrm{C}$ and the supernatants were used for the enzyme assay. Briefly, $5 \mu$ of plasma or brain extract were added to the reaction mixture in the wells of a 96 well assay plate and the increase in optical density at 400 $\mathrm{nm}$ was measured every minute up to 5 min using SpectraMax M5 spectrophotometer (Molecular Devices, Sunnyvale, CA). The increase in optical density per minute was used for determination of enzyme activity.

\subsection{Western blot analysis of brain homogenates}

Brain homogenates $(10 \% \mathrm{w} / \mathrm{v})$ were prepared in T-PER tissue protein extraction buffer (Pierce Chemical Co, Rockford, IL) containing protease and phosphatase inhibitor cocktails (Sigma-Aldrich, St. Louis, MO) using an ultrasonic homogenizer. The homogenates were centrifuged at $5000 \times g$ for $5 \mathrm{~min}$ at $4^{\circ} \mathrm{C}$ and $2 \mu \mathrm{l}$ each of supernatants were used for Western blotting. Rabbit polyclonal antibodies, which bind specifically to phosphorylated serine396 (S396) of Tau protein, were purchased from Abcam (Cambridge, MA) and used at dilution of $1: 5,000$. Mouse monoclonal antibodies against TNAP and $\beta$-actin were obtained from Sigma-Aldrich (St. Louis, MO) and used at a dilution of $1: 2,000$ and $1: 40,000$ respectively. Secondary antibodies 
labeled with horse-radish peroxidase (HRP) was purchased from Santa Cruz Biotechnology (Santa Cruz, CA) and used at a dilution of $1: 2,500$. SDS-polyacrylamide gel electrophoresis and Western blotting analysis were carried out as previously described by us earlier [0]. After Western blotting, the protein bands were detected using ECL-Plus Western blot detection reagent (GE Healthcare, Piscataway, NJ) and the chemiluminescence was imaged in an Alphalmage reader (Cell Biosciences, Santa Clara, CA). The band intensity was measured by densitometry using AlphaView software (Cell Biosciences, Santa Clara, CA).

\subsection{Statistical analysis}

Statistical analysis was carried out by Repeated Measures Analysis of Variance (ANOVA) followed by Tukey's post-hoc test using HSD multiple comparisons (SPSS software, Version 20). Values were expressed as mean \pm standard deviation (SD). A $p$ value less than 0.05 was considered significant.

\section{RESULTS}

\subsection{Expression of pTau in brain regions after injury}

Western blot analyses of extracts from the different brain regions after either blast or weight drop showed significant increases in the expression of pTau (S396) at 6 and $24 \mathrm{hr}$ post-insult (Figure 1), with increases ranging from 23 to $34 \%$. At $6 \mathrm{hr}$, the ratios (mean $\pm S D$ ) of band intensities of $p$ Taulactin for sham, blast and weight drop were $0.88 \pm 0.06,1.24 \pm 0.17$ and $1.34 \pm 0.23$ respectively in the brain stem, $0.74 \pm 0.02$, $0.97 \pm 0.11$ and $1.12 \pm 0.21$ respectively in the hippocampus, and $0.79 \pm 0.05,1.03 \pm 0.15$ and $1.15 \pm 0.02$ respectively in the cortex. At $24 \mathrm{hr}$, the ratios for sham, blast and weight drop were $0.84 \pm 0.09,1.09 \pm 0.09$ and $1.04 \pm 0.06$ respectively in the brain stem, 
$0.76 \pm 0.05,0.86 \pm 0.02$ and $0.87 \pm 0.03$ respectively in the hippocampus, and $0.78 \pm 0.08$ $0.96 \pm 0.01$ and $0.97 \pm 0.01$ respectively in the cortex. Although the increases resulting from the two different insults were not significantly different at these respective injury severities, the elevated levels of pTau in different brain regions of animals exposed to blast overpressure were generally less than those seen in rats subjected to weight drop injury. The levels of pTau in different brain regions were similar $24 \mathrm{hr}$ after either insult and were significantly greater than measured in the sham controls. We have detected two adjacent bands in the Western blot at the expected position of $p T a u$, which could be due to the difference in the number of amino acids phosphorylated or due to other posttranslational modifications to Tau protein after injury. For densitometry analysis, the intensities of both bands were used.

\subsection{Activity of TNAP in brain regions after injury}

Figure 2 shows the activity of TNAP in different brain regions at 6 and $24 \mathrm{hr}$ after blast or weight drop induced brain injury. At $6 \mathrm{hr}$, the TNAP activity (units/mg protein) for sham, blast and weight drop were $214.14 \pm 62.81,119.20 \pm 16.66$ and $106.86 \pm 17.29$ respectively in the brain stem, $173.84 \pm 47.69,118.31 \pm 19.24$ and $107.21 \pm 22.23$ respectively in the hippocampus, $209.07 \pm 116.54,144.29 \pm 28.38$ and $125.51 \pm 30.92$ respectively in the cortex. At $24 \mathrm{hr}$, the TNAP activity for sham, blast and weight drop were $206.88 \pm 17.48,166.02 \pm 23.17$ and $159.58 \pm 18.25$ respectively in the brain stem, $181.52 \pm 28.44,140.93 \pm 11.56$ and $133.80 \pm 22.52$ respectively in the hippocampus, $203.18 \pm 20.59,168.08 \pm 16.82$ and $158.07 \pm 20.80$ respectively in the cortex. Both insults significantly decreased the activity of TNAP at both 6 and $24 \mathrm{hr}$ post-injury. At $6 \mathrm{hr}$ postinjury, blast exposure decreased TNAP activity in brainstem, hippocampus, and cortex 
by $45 \%, 32 \%$ and $31 \%$ respectively, whereas weight drop caused regional decreases of $51 \%, 39 \%$ and $40 \%$ respectively. At $24 \mathrm{hr}$, TNAP activity in brainstem, hippocampus and cortex remained decreased by $20 \%, 23 \%$ and $18 \%$, respectively after blast whereas after weight drop, the decreases were $23 \%, 27 \%$ and $23 \%$ respectively. Although once again the changes resulting from the two insults were not significantly different, at these respective injury severities, weight drop consistently caused slightly greater decreases in TNAP activity than were seen after blast exposure, which were greater at $6 \mathrm{~h}$ than at $24 \mathrm{hr}$ post-injury

Activity of alkaline phosphatase in the plasma

The baseline activities of alkaline phosphatase in the plasma for sham, blast and weight drop groups were $101.45 \pm 15.27,97.61 \pm 16.72$ and $94.05 \pm 10.97$ respectively. At $6 \mathrm{hr}$, the activities of alkaline phosphatase in the plasma for sham, blast and weight drop group were $98.43 \pm 19.78,88.56 \pm 19.58$ and $63.61 \pm 3.85$ respectively. At $24 \mathrm{hr}$, the activities of alkaline phosphatase in the plasma for sham, blast and weight drop group were $90.48 \pm 4.07,83.08 \pm 6.14$ and $59.50 \pm 5.30$ respectively. Total alkaline phosphatase activity in the plasma decreased significantly at 6 and $24 \mathrm{hr}$ after weight drop, in contrast to blast exposure, which did not significantly alter activity at either time postinjury (Figure 3). Weight drop caused $32 \%$ and $37 \%$ decreases in TNAP activity in the plasma at 6 and $24 \mathrm{hr}$ respectively, yielding significantly different measurements than were seen in sham and blast-injured rats at these times.

3.3. Expression of TNAP in the brain regions after blast and weight drop

Figure 4 shows the expression of TNAP in different brain regions at 6 and $24 \mathrm{hr}$ post-injury. At $6 \mathrm{hr}$, the ratios (mean $\pm \mathrm{SD}$ ) of band intensities of TNAP/actin for sham, 
blast and weight drop were $0.84 \pm 0.06,0.70 \pm 0.04$ and $0.64 \pm 0.07$ respectively in the brain stem, $0.81 \pm 0.04,0.69 \pm 0.05$ and $0.65 \pm 0.06$ respectively in the hippocampus, $0.76 \pm 0.11,0.60 \pm 0.01$ and $0.59 \pm 0.03$ respectively in the cortex. At $24 \mathrm{hr}$, the ratios for sham, blast and weight drop were $0.80 \pm 0.02,0.72 \pm 0.03$ and $0.71 \pm 0.04$ respectively in the brain stem, $0.77 \pm 0.05,0.69 \pm 0.02$ and $0.69 \pm 0.01$ respectively in the hippocampus, $0.74 \pm 0.01,0.68 \pm 0.03$ and $0.67 \pm 0.02$ respectively in the cortex. Compared to sham controls, both blast and weight drop significantly decreased the expression of TNAP at 6 and $24 \mathrm{hr}$ post-injury. The magnitudes of the changes in different brain regions, ranged from 17 to $30 \%$, were quite comparable after either insult and were generally greater at 6 than at $24 \mathrm{hr}$ post-injury.

\subsection{Expression of APP in the brain regions after injury}

The expression of APP in brain regions at $6 \& 24 \mathrm{hr}$ after blast or weight drop is shown in Figure 5. At $6 \mathrm{hr}$, the ratios (mean $\pm \mathrm{SD}$ ) of band intensities of APP/actin for sham, blast and weight drop were $0.99 \pm 0.10,1.18 \pm 0.27$ and $1.09 \pm 0.19$ respectively in the brain stem, $0.94 \pm 0.07,1.11 \pm 0.16$ and $1.05 \pm 0.18$ respectively in the hippocampus, $0.87 \pm 0.07,1.01 \pm 0.16$ and $0.98 \pm 0.16$ respectively in the cortex. At $24 \mathrm{hr}$, the ratios for sham, blast and weight drop were $1.02 \pm 0.06,1.51 \pm 0.27$ and $1.25 \pm 0.12$ respectively in the brain stem, $0.93 \pm 0.02,1.16 \pm 0.12$ and $1.00 \pm 0.02$ respectively in the hippocampus, $0.86 \pm 0.03,1.18 \pm 0.02$ and $1.00 \pm 0.08$ respectively in the cortex. The expression of APP in different brain regions was increased significantly after blast and weight drop compared to sham controls. Despite any statistical significance, the increased expression of APP in the brain regions was more after blast exposure compared to weight drop. 


\section{DISCUSSION}

In this study, we report for the first time that brain injury causes an acute decrease in the protein level and activity of TNAP in the brain which is associated with an acute increase in the phosphorylation of Tau protein. In view of the previously reported role of this enzyme in the dephosphorylation of $p$ Tau protein, the acutely diminished level and lactivity of TNAP after injury might promote the progressive accumulation of $p T a u$ as an early event leading to eventual tauopathy. Neither decreased TNAP activity nor increased accumulation of $p$ Tau in the brain after injury correlated closely with the deposition of APP, suggesting a distinct mechanism of tauopathy after brain injury compared to $A D$.

There are at least four distinct forms of alkaline phosphatase enzyme present in mammals - intestinal, placental, placental-like and TNAP (present in liver, bone, kidney, brain etc.). TNAP is sequenced in chromosome 1 and sequences for all other isozymes are located in chromosome 2. In the brain, several roles for TNAP have been reported, which include: (1) involvement in the development of the nervous system partly as an ectonucleotidase in neurogenic zones [16], (2) participation in neuronal migration by interaction with collagen $[3,21],(3)$ regulation of the synthesis of neurotransmitters such as $\mathrm{Y}$-aminobutyric acid, dopamine and serotonin [22] and (4) dephosphorylation of $p$ Tau $[11,14,30]$. As described in the introduction, TNAP is the principal protein phosphatase enzyme present in the brain that is primarily involved in the dephosphorylation of $p T a u$. Although other protein phosphatases such as PP-1, 2A, 2B and 2C can also dephosphorylate $p$ Tau, TNAP has been shown to have higher activity towards $p$ Tau than these other phosphatases [30]; hence, a decrease in TNAP level/activity alone can 
result in significant accumulation of $p T a u$. Non-phosphorylated Tau protein is essential for microtubule assembly with tubulin and accumulation of $p$ Tau disrupts this process, resulting in neuronal hypofunction and even neuronal death.

The phosphorylation of Tau protein can occur on different amino acids at specific locations of the amino acid sequence. Tau protein, phosphorylated at serine396 (S396), is rich in the paired helical filaments which form neurofibrillary tangles observed in the brains of patients with $A D[2,12,34]$. Our results showed increased phosphorylation of S396 after blast and weight drop-induced brain injuries, pointing to the possibility of formation of neurofibrillary tangles after brain injury. Increased phosphorylation of Tau protein at S396 at $24 \mathrm{hr}$ after blast exposure has been previously reported [13], supporting our current observation. In addition to S396, increased phosphorylation of several other amino acids in the Tau protein was observed in that study which could potentially also contribute to disrupted microtubule assembly after blast exposure. Using head impact-induced single and repetitive mild TBI in $h$ Tau mice, Ojo et. al. showed that repetitive, but not single, mild TBI also induced the phosphorylation of Tau at a number of amino acid sites [24]. The phosphorylation of Tau protein after single head impact in the current study could simply reflect a more severe insult in these subjects than was produced by a single impact in this earlier work.

In the present study, we have shown that the protein levels and activities of TNAP in the brain decreased significantly after blast exposure or impact-acceleration and were associated with a significant increase in the phosphorylation of Tau protein. At these respective injury severities, the decreases in TNAP activity were greater after weight drop than after blast and were concomitantly associated with comparably greater 
increases in the levels of $p T a u$, suggesting that decreased TNAP activity may in part be responsible for the accumulation of $p$ Tau after these brain insults. If persistent, decreased TNAP activity might promote progressive accumulation of $p$ Tau, culminating in tauopathy and conditions associated with chronic diseases such as CTE. Long-term studies evaluating the level/activity of TNAP and the accumulation of $p$ Tau in the brain are warranted to determine their roles in the development of chronic diseases. The decrease in the activity of the enzyme could be due to the decreased level of the protein or due to an inhibition of the enzyme activity. Western blot analyses of extracts from the three brain regions indicate that the TNAP protein level consistently decreased after the brain insults. The decreased protein level of TNAP in the brain regions could be due to decreased synthesis or increased degradation after injury, and further studies using messenger RNA levels are warranted to delineate the underlying mechanism.

The major alkaline phosphatase isozyme present in the blood is TNAP [23], and the decrease in the activity of TNAP in the brain was associated with a significant decrease in the activity of total alkaline phosphatase in the plasma after weight drop but not after blast exposure. Although it is not clear why plasma alkaline phosphatase activity declines after weight drop, this parallel with the brain points to the possibility that the two changes are related and that phosphatase activity in the circulation might be attributed to TNAP enzyme originating in the brain, which is diminished after injury. Unlike weight drop, where injury is localized to the brain, blast is a whole body insult affecting multiple organ systems; consequently, changes in total alkaline phosphatase activity in the circulation after blast may more generally reflect the net outcome of its composite effects on multiple organ systems and not just brain. In particular, since blast 
exposure disrupts cell membranes in various organs resulting in the release of enzymes and other intracellular components $[0,1,31]$, it is possible that unlike weight drop injury, blast exposure causes the release/leakage of different alkaline phosphatase isozymes into the plasma from peripheral organs such as liver, kidney, intestine, placenta, bone etc., all of which contribute to the alkaline phosphatase activity measured in plasma after blast exposure.

TNAP activity in the plasma has been reported to increase after 7 days postinjury in patients with different brain injury conditions such as post-resuscitation encephalopathy, ruptured cerebral aneurysms, subdural hematoma, cerebral contusion and non-traumatic intracerebral hemorrhage [35]. Although no change in TNAP activity was observed in those patients at early stages of injury, patients who died at early stages of injury had very low or undetectable TNAP activity in their plasma, which is in agreement with our observation of low TNAP activity at acute stages of injury.

In $A D$, the accumulation of amyloid plaques have been reported to precede and promote tauopathy $[10,17,26,27,29,33]$. In particular, formation of amyloid $\beta$ (Aß) protein has been shown to enhance the phosphorylation of Tau protein, resulting in neurofibrillary tangle formation in an APP and Tau double transgenic mouse model [27]. The phosphorylation of Tau protein has been further proposed to involve the activation of mitogen-activated protein kinase by accumulated APP or A $\beta$ protein [10]. In contrast, previous shock tube studies with rats revealed a divergence in the changes of these proteins in brain after blast exposure. Whereas the expression of APP increased in the brain at $24 \mathrm{hr}$ after blast and increased further after 1 week, the levels of $A \beta$ peptides progressively decreased during this time [8], indicating that increased accumulation of 
$A \beta$ peptides may not trigger the phosphorylation of Tau protein after blast exposure. In the present study, comparison of the accumulation of pTau (S396) in brain after weight drop and blast injury (Fig.1) with the expression of APP after these insults (Fig.5), reveals a similar divergence in these measurements, reinforcing interpretation that accumulated APP may not play a substantial role promoting the phosphorylation of Tau protein after blast exposure. Blast or weight drop didn't increase the expression of APP in the brain at $6 \mathrm{hr}$ post-injury. At $24 \mathrm{hr}$, both insults significantly increased expression of APP, but the greatest increase in APP was observed after blast exposure (Fig.5) unlike pTau, where the greatest increase was evoked by weight drop (Fig.1). Although these disparities do not rule out APP promoting phosphorylation of Tau, they nevertheless do raise the possibility that the neurobiological mechanisms yielding tauopathy after TBI might be distinct from those of $A D$.

\section{CONCLUSION}

Brain injury after blast exposure or weight drop results in a significant decrease in the expression and activity of TNAP which is associated with a significant increase in the accumulation of pTau in different brain regions. In view of the known function of TNAP in dephosphorylating $p T a u$, the accumulation of $p T a u$ after brain injury could be attributed to the decreased TNAP activity/levels in the brain after the injury. The decreased activity of TNAP in the brain after injury was associated with a significantly decreased total alkaline phosphatase activity in the plasma. The accumulation of $p T a u$ after brain injury did not show a positive correlation with the expression of APP in different brain regions, indicating that the mechanism of tauopathy after brain injury could be distinct from that of $A D$. 


\section{ACKNOWLEDGEMENTS}

Technical help received from Rania Abu-Taleb, Andrea Edwards and Cory Riccio is greatly acknowledged. This work was supported by Congressionally Directed Medical Research Program awards W81XWH-08-2-0018 and W81XWH-11-2-0127 to JBL.

\section{REFERENCES}

[0] P. Arun, R. Abu-Taleb, S. Oguntayo, M. Tanaka, Y. Wang, M. Valiyaveettil, J.B. Long, Y. Zhang, M.P. Nambiar, Distinct patterns of expression of traumatic brain injury biomarkers after blast exposure: role of compromised cell membrane integrity, Neuroscience letters 552 ( 2013) 87-91.

[1] P. Arun, S. Oguntayo, Y. Alamneh, C. Honnold, Y. Wang, M. Valiyaveettil, J.B. Long, M.P. Nambiar, Rapid release of tissue enzymes into blood after blast exposure: potential use as biological dosimeters, PloS one 7 ( 2012) e33798.

[2] J. Bertrand, V. Plouffe, P. Senechal, N. Leclerc, The pattern of human tau phosphorylation is the result of priming and feedback events in primary hippocampal neurons, Neuroscience 168 ( 2010) 323-334.

[3] M. Bossi, M.F. Hoylaerts, J.L. Millan, Modifications in a flexible surface loop modulate the isozyme-specific properties of mammalian alkaline phosphatases, The Journal of biological chemistry 268 ( 1993) 25409-25416.

[4] J. Busciglio, A. Lorenzo, J. Yeh, B.A. Yankner, beta-amyloid fibrils induce tau phosphorylation and loss of microtubule binding, Neuron 14 ( 1995) 879-888.

[5] E. Calabrese, F. Du, R.H. Garman, G.A. Johnson, C. Riccio, L.C. Tong, J.B. Long, Diffusion tensor imaging reveals white matter injury in a rat model of repetitive blast-induced traumatic brain injury, Journal of neurotrauma 31 ( 2014) 938-950.

[6] I. Cernak, A.C. Merkle, V.E. Koliatsos, J.M. Bilik, Q.T. Luong, T.M. Mahota, L. Xu, N. Slack, D. Windle, F.A. Ahmed, The pathobiology of blast injuries and 
blast-induced neurotrauma as identified using a new experimental model of injury in mice, Neurobiol Dis 41 ( 2011) 538-551.

[7] I. Cernak, Z. Wang, J. Jiang, X. Bian, J. Savic, Ultrastructural and functional characteristics of blast injury-induced neurotrauma, J Trauma 50 ( 2001) 695706.

[8] R. De Gasperi, M.A. Gama Sosa, S.H. Kim, J.W. Steele, M.C. Shaughness, E. Maudlin-Jeronimo, A.A. Hall, S.T. Dekosky, R.M. McCarron, M.P. Nambiar, S. Gandy, S.T. Ahlers, G.A. Elder, Acute blast injury reduces brain abeta in two rodent species, Frontiers in neurology 3 ( 2012) 177.

[9] L.E. Goldstein, A.M. Fisher, C.A. Tagge, X.L. Zhang, L. Velisek, J.A. Sullivan, C. Upreti, J.M. Kracht, M. Ericsson, M.W. Wojnarowicz, C.J. Goletiani, G.M. Maglakelidze, N. Casey, J.A. Moncaster, O. Minaeva, R.D. Moir, C.J. Nowinski, R.A. Stern, R.C. Cantu, J. Geiling, J.K. Blusztajn, B.L. Wolozin, T. Ikezu, T.D. Stein, A.E. Budson, N.W. Kowall, D. Chargin, A. Sharon, S. Saman, G.F. Hall, W.C. Moss, R.O. Cleveland, R.E. Tanzi, P.K. Stanton, A.C. McKee, Chronic traumatic encephalopathy in blast-exposed military veterans and a blast neurotrauma mouse model, Sci Transl Med 4 ( 2012) 134ra160.

[10] S.M. Greenberg, E.H. Koo, D.J. Selkoe, W.Q. Qiu, K.S. Kosik, Secreted betaamyloid precursor protein stimulates mitogen-activated protein kinase and enhances tau phosphorylation, Proc Natl Acad Sci U S A 91 ( 1994) 71047108.

[11] D.P. Hanger, J.P. Brion, J.M. Gallo, N.J. Cairns, P.J. Luthert, B.H. Anderton, Tau in Alzheimer's disease and Down's syndrome is insoluble and abnormally phosphorylated, Biochem J 275 ( Pt 1) ( 1991) 99-104.

[12] D.P. Hanger, K. Hughes, J.R. Woodgett, J.P. Brion, B.H. Anderton, Glycogen synthase kinase-3 induces Alzheimer's disease-like phosphorylation of tau: generation of paired helical filament epitopes and neuronal localisation of the kinase, Neuroscience letters 147 ( 1992) 58-62.

[13] B.R. Huber, J.S. Meabon, T.J. Martin, P.D. Mourad, R. Bennett, B.C. Kraemer, I. Cernak, E.C. Petrie, M.J. Emery, E.R. Swenson, C. Mayer, E. Mehic, E.R. 
Peskind, D.G. Cook, Blast exposure causes early and persistent aberrant phospho- and cleaved-tau expression in a murine model of mild blast-induced traumatic brain injury, Journal of Alzheimer's disease : JAD 37 ( 2013) 309323.

[14] K. Iqbal, T. Zaidi, C. Bancher, I. Grundke-lqbal, Alzheimer paired helical filaments. Restoration of the biological activity by dephosphorylation, FEBS Lett 349 ( 1994) 104-108.

[15] J.D. Kocsis, A. Tessler, Pathology of blast-related brain injury, J Rehabil Res Dev 46 ( 2009) 667-672.

[16] D. Langer, Y. Ikehara, H. Takebayashi, R. Hawkes, H. Zimmermann, The ectonucleotidases alkaline phosphatase and nucleoside triphosphate diphosphohydrolase 2 are associated with subsets of progenitor cell populations in the mouse embryonic, postnatal and adult neurogenic zones, Neuroscience 150 ( 2007) 863-879.

[17] W.D. Le, W.J. Xie, R. Kong, S.H. Appel, Beta-amyloid-induced neurotoxicity of a hybrid septal cell line associated with increased tau phosphorylation and expression of beta-amyloid precursor protein, Journal of neurochemistry 69 ( 1997) 978-985.

[18] J.B. Long, T.L. Bentley, K.A. Wessner, C. Cerone, S. Sweeney, R.A. Bauman, Blast overpressure in rats: recreating a battlefield injury in the laboratory, Journal of neurotrauma 26 ( 2009) 827-840.

[19] J. Magnuson, F. Leonessa, G.S. Ling, Neuropathology of explosive blast traumatic brain injury, Curr Neurol Neurosci Rep 12 ( 2012) 570-579.

[20] A. Marmarou, M.A. Foda, W. van den Brink, J. Campbell, H. Kita, K. Demetriadou, A new model of diffuse brain injury in rats. Part I: Pathophysiology and biomechanics, Journal of neurosurgery 80 ( 1994) 291-300.

[21] S. Narisawa, H. Hasegawa, K. Watanabe, J.L. Millan, Stage-specific expression of alkaline phosphatase during neural development in the mouse, Developmental dynamics : an official publication of the American Association of Anatomists 201 ( 1994) 227-235. 
[22] L. Negyessy, J. Xiao, O. Kantor, G.G. Kovacs, M. Palkovits, T.P. Doczi, L. Renaud, G. Baksa, T. Glasz, M. Ashaber, P. Barone, C. Fonta, Layer-specific activity of tissue non-specific alkaline phosphatase in the human neocortex, Neuroscience 172 ( 2011) 406-418.

[23] Y. Nishihara, Y. Hayashi, T. Fujii, T. Adachi, T. Stigbrand, K. Hirano, The alkaline phosphatase in human plexus chorioideus, Biochimica et biophysica acta 1209 ( 1994) 274-278.

[24] J.O. Ojo, B. Mouzon, M.B. Greenberg, C. Bachmeier, M. Mullan, F. Crawford, Repetitive mild traumatic brain injury augments tau pathology and glial activation in aged hTau mice, Journal of neuropathology and experimental neurology 72 ( 2013) 137-151.

[25] A. Saljo, F. Bao, J. Shi, A. Hamberger, H.A. Hansson, K.G. Haglid, Expression of c-Fos and c-Myc and deposition of beta-APP in neurons in the adult rat brain as a result of exposure to short-lasting impulse noise, Journal of neurotrauma 19 ( 2002) 379-385.

[26] E. Samura, M. Shoji, T. Kawarabayashi, A. Sasaki, E. Matsubara, T. Murakami, X. Wuhua, S. Tamura, M. Ikeda, K. Ishiguro, T.C. Saido, D. Westaway, P. St George Hyslop, Y. Harigaya, K. Abe, Enhanced accumulation of tau in doubly transgenic mice expressing mutant betaAPP and presenilin-1, Brain research 1094 ( 2006) 192-199.

[27] Y. Seino, T. Kawarabayashi, Y. Wakasaya, M. Watanabe, A. Takamura, Y. Yamamoto-Watanabe, T. Kurata, K. Abe, M. Ikeda, D. Westaway, T. Murakami, P.S. Hyslop, E. Matsubara, M. Shoji, Amyloid beta accelerates phosphorylation of tau and neurofibrillary tangle formation in an amyloid precursor protein and tau double-transgenic mouse model, Journal of neuroscience research 88 ( 2010) 3547-3554.

[28] S.I. Svetlov, V. Prima, D.R. Kirk, H. Gutierrez, K.C. Curley, R.L. Hayes, K.K. Wang, Morphologic and biochemical characterization of brain injury in a model of controlled blast overpressure exposure, J Trauma 69 ( 2010) 795-804. 
[29] Y. Tomidokoro, K. Ishiguro, Y. Harigaya, E. Matsubara, M. Ikeda, J.M. Park, K. Yasutake, T. Kawarabayashi, K. Okamoto, M. Shoji, Abeta amyloidosis induces the initial stage of tau accumulation in APP( Sw) mice, Neuroscience letters 299 ( 2001) 169-172.

[30] J.Z. Wang, I. Grundke-lqbal, K. Iqbal, Restoration of biological activity of Alzheimer abnormally phosphorylated tau by dephosphorylation with protein phosphatase-2A, -2B and -1, Brain Res Mol Brain Res 38 ( 1996) 200-208.

[31] Y. Wang, P. Arun, Y. Wei, S. Oguntayo, R. Gharavi, M. Valiyaveettil, M.P. Nambiar, J.B. Long, Repeated blast exposures cause brain DNA fragmentation in mice, Journal of neurotrauma 31 ( 2014) 498-504.

[32] Y. Wang, Y. Wei, S. Oguntayo, W. Wilkins, P. Arun, M. Valiyaveettil, J. Song, J.B. Long, M.P. Nambiar, Tightly coupled repetitive blast-induced traumatic brain injury: development and characterization in mice, Journal of neurotrauma 28 ( 2011) 2171-2183.

[33] Z.F. Wang, H.L. Li, X.C. Li, Q. Zhang, Q. Tian, Q. Wang, H. Xu, J.Z. Wang, Effects of endogenous beta-amyloid overproduction on tau phosphorylation in cell culture, Journal of neurochemistry 98 ( 2006) 1167-1175.

[34] D. Xia, C. Li, J. Gotz, Pseudophosphorylation of Tau at distinct epitopes or the presence of the P301L mutation targets the microtubule-associated protein Tau to dendritic spines, Biochimica et biophysica acta 1852 ( 2015) 913-924.

[35] M. Yamashita, M. Sasaki, K. Mii, M. Tsuzuki, K. Takakura, S. Yoshinoya, A. Ohkubo, Measurement of serum alkaline phosphatase isozyme I in braindamaged patients, Neurologia medico-chirurgica 29 ( 1989) 995-998. 


\section{Figure Legends}

Figure 1. (A) Western blotting showing the expression of $p T a u$, with phosphorylation at serine396, in different brain regions at $6 \mathrm{hr}$ and $24 \mathrm{hr}$ after blast or weight drop. The antibody detects Tau protein only when the serine396 is phosphorylated.

Representative figures from two rats out of four in each group are shown. (B)

Densitometry analysis showing the ratio of band intensities of $p$ Tau and $\beta$-actin. Values are expressed as mean $\pm \mathrm{SD}$. ${ }^{*} p<0.05$.

Figure 2. Activity of TNAP in different brain regions at $6 \mathrm{hr}$ and $24 \mathrm{hr}$ after blast or weight drop. One unit of enzyme activity is the $\mu$ moles of $p$-nitrophenol liberated per minute.

Values are expressed as mean $\pm \mathrm{SD} . \mathrm{n}=4,{ }^{*} p<0.05,{ }^{* *} p<0.01$.

Figure 3. Activity of alkaline phosphatase in the plasma at different intervals after blast or weight drop. One unit of enzyme activity is the $\mu$ moles of $p$-nitrophenol liberated per minute. Baseline - plasma collected before injury. Values are expressed as mean \pm SD. $\mathrm{n}=4,{ }^{*} p<0.05,{ }^{* *} p<0.01$.

Figure 4. (A) Western blotting showing the expression of TNAP in different brain regions at $6 \mathrm{hr}$ and $24 \mathrm{hr}$ after blast or weight drop. Representative figures from two rats out of four in each group are shown. (B) Densitometry analysis showing the ratio of band intensities of TNAP and $\beta$-actin. Values are expressed as mean \pm SD. ${ }^{*} p<0.05$.

Figure 5. (A) Western blotting showing the expression of APP in different brain regions at 6 and $24 \mathrm{hr}$ after blast or weight drop. Representative figures from two rats out of four in each group are shown. (B) Densitometry analysis showing the ratio of band intensities of APP and $\beta$-actin. Values are expressed as mean \pm SD. ${ }^{*} p<0.05$. 
(A)

Fig. 1
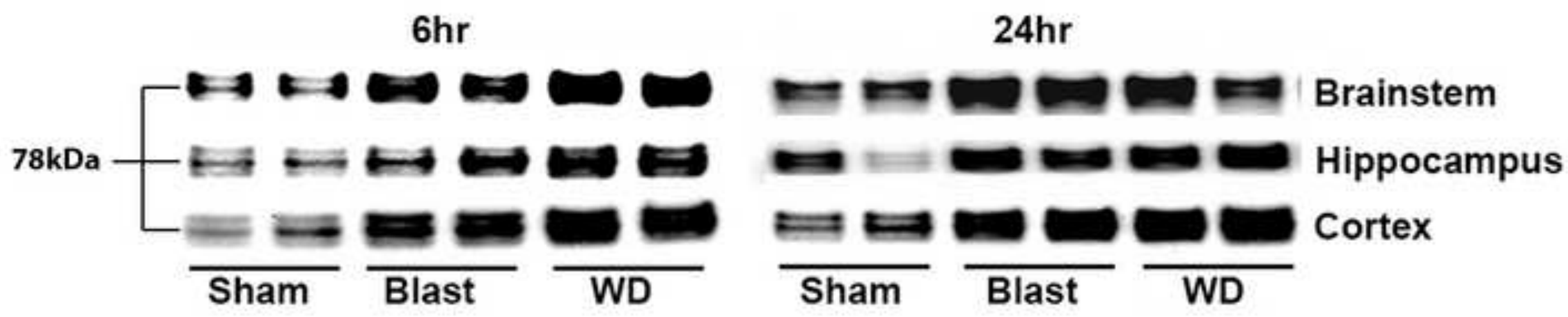

(B)
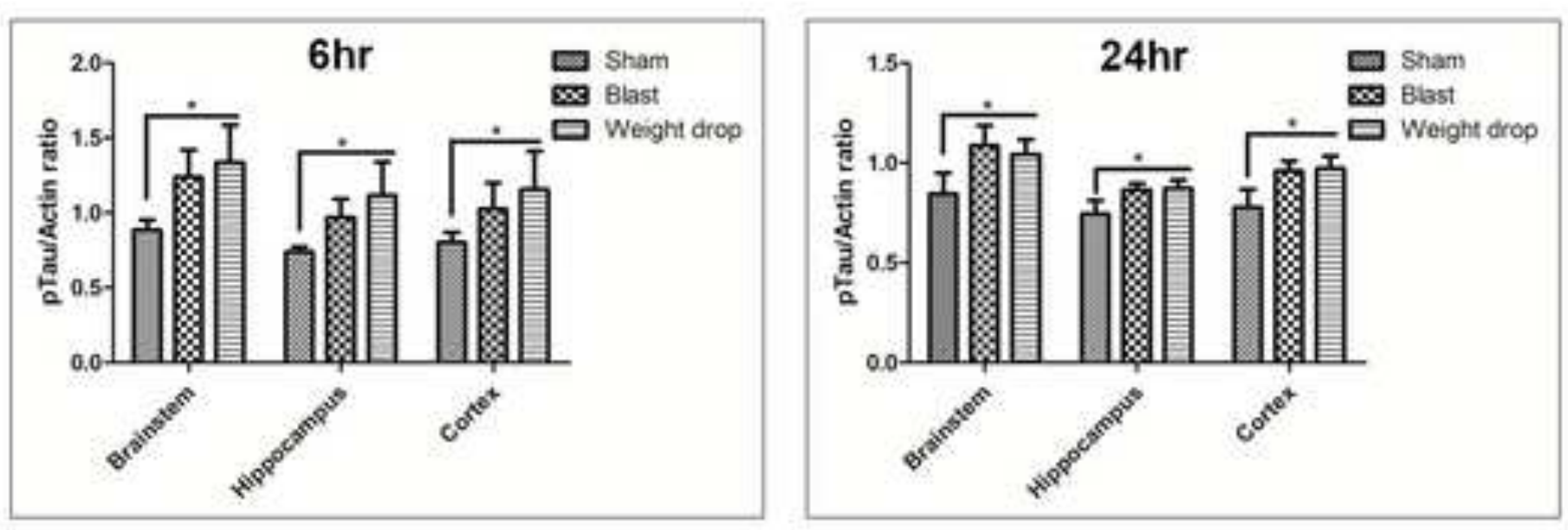
Fig.2
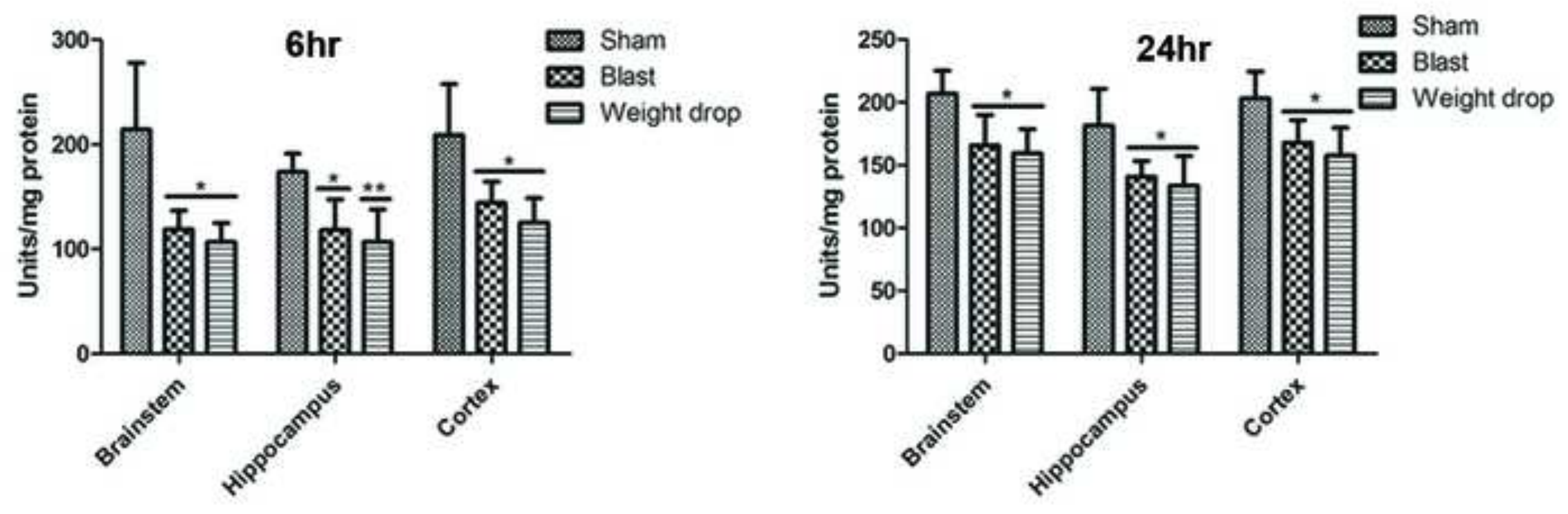
Fig.3

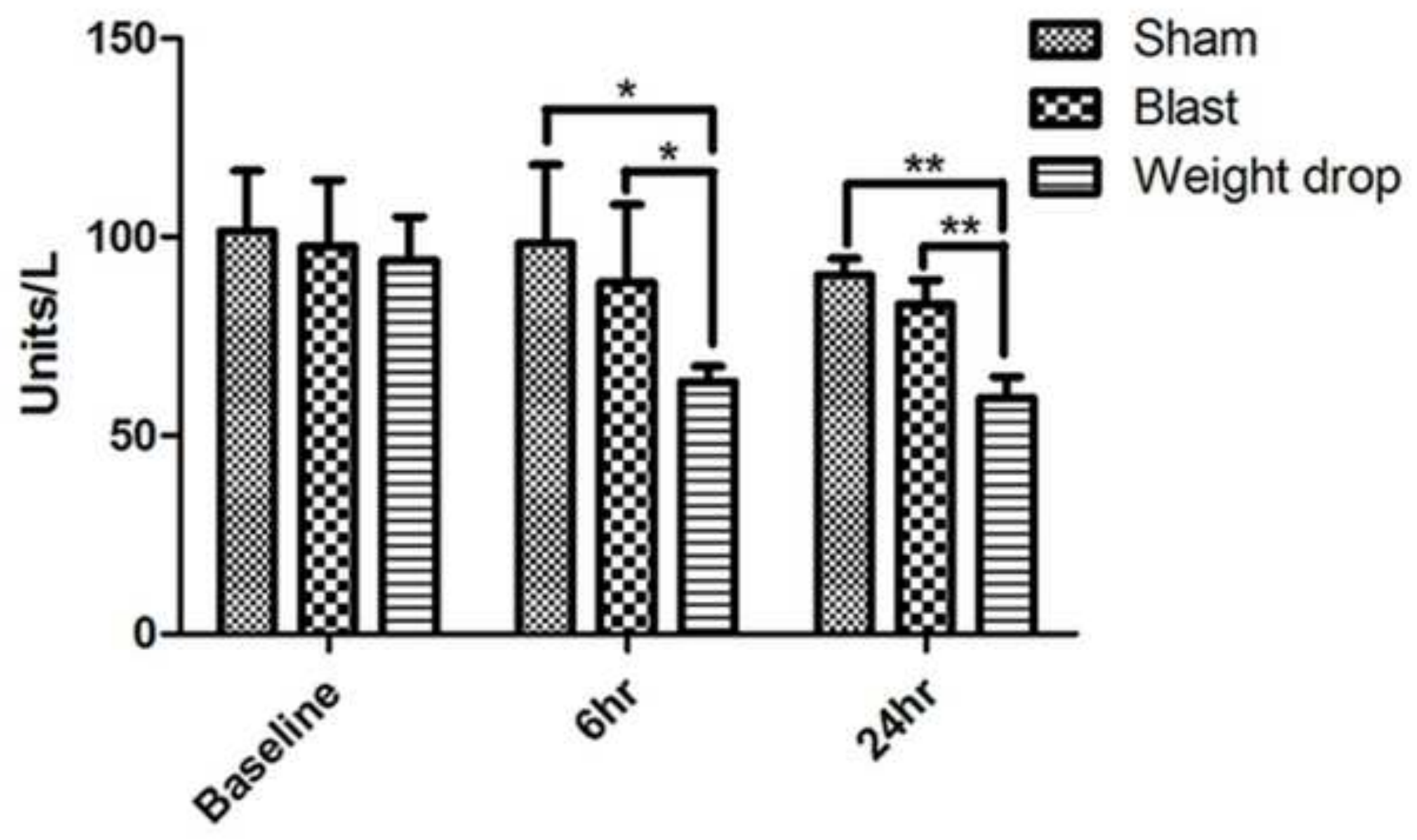


Fig. 4

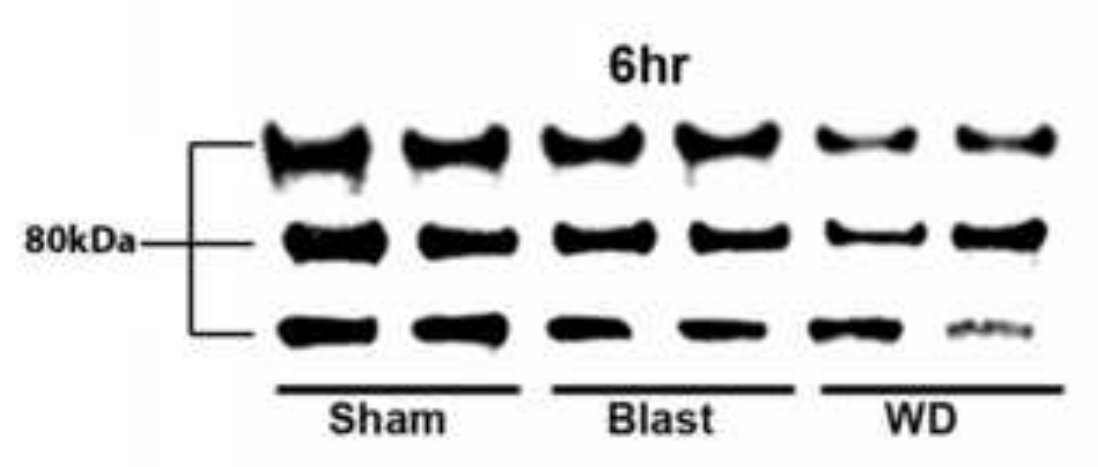

(A)

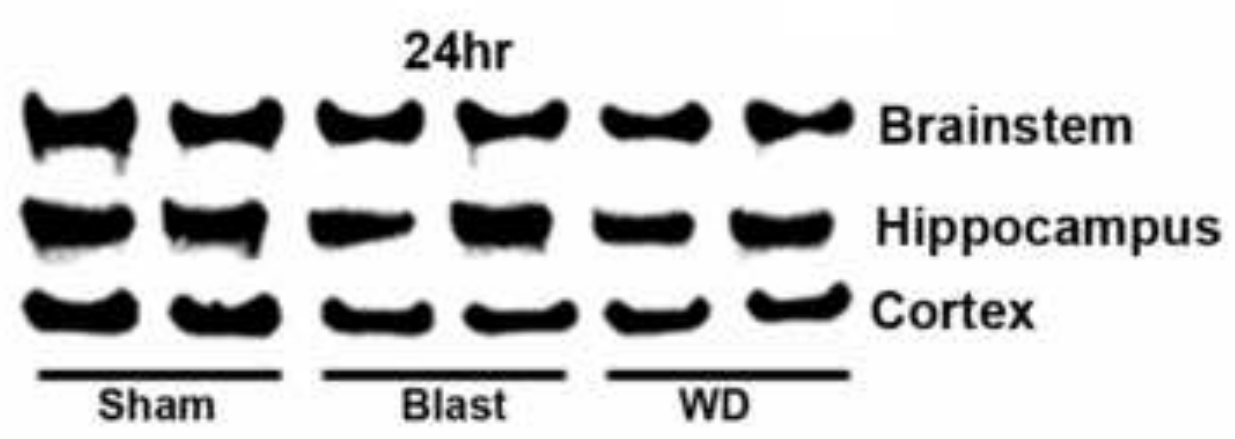

(B)
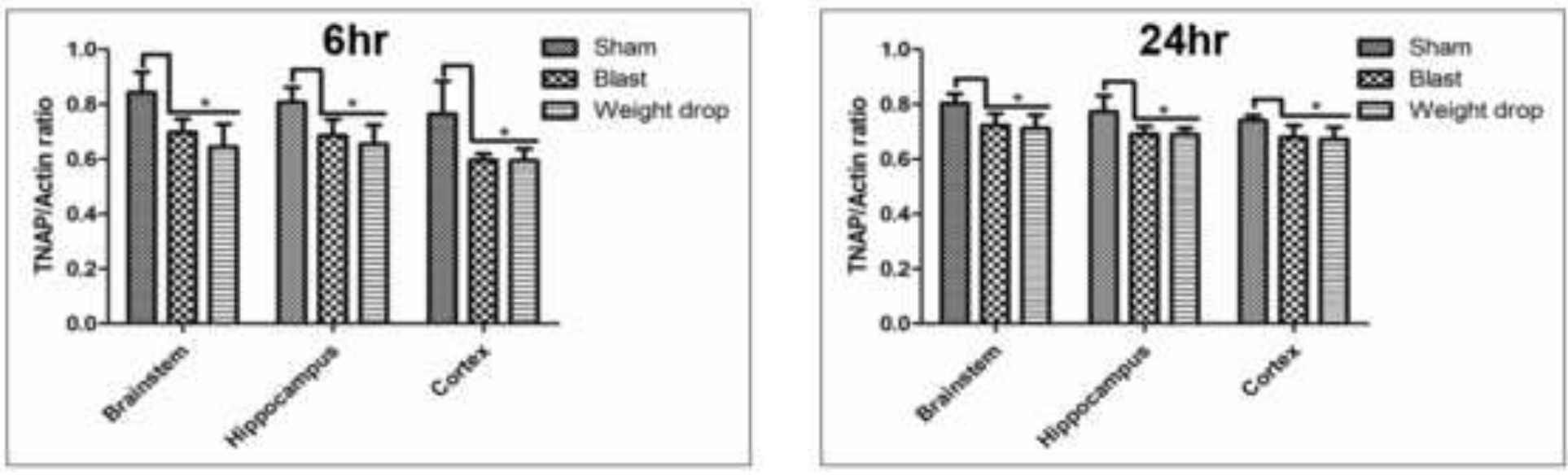
Fig.5

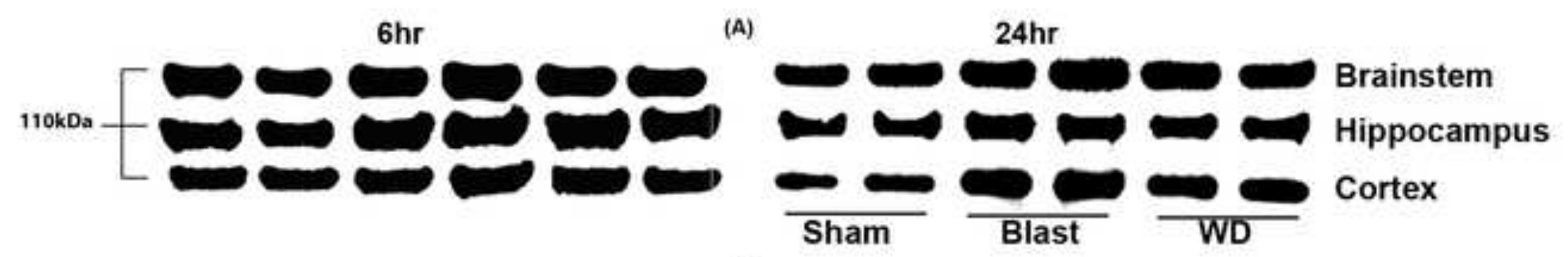

(B)
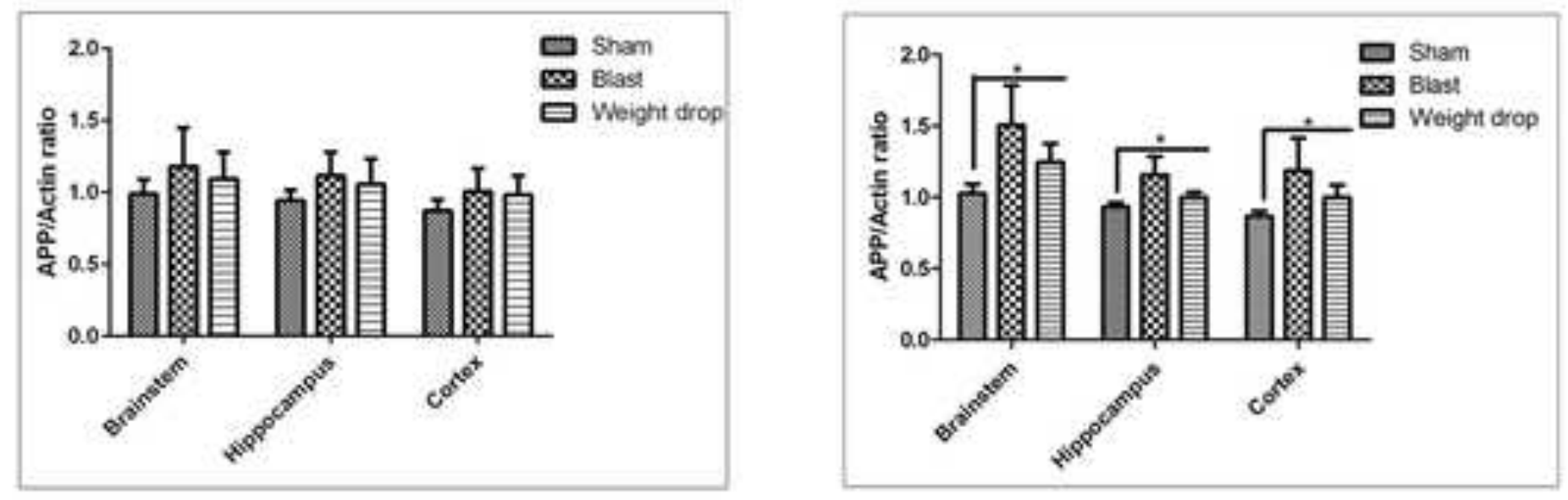